\title{
Víctimas del desplazamiento forzado: comorbilidad entre trastorno por estrés postraumático (TEPT) y depresivo mayor (TDM)
}

\author{
Yury Maciel Torres Salazar \\ Psicóloga \\ Universidad Pontificia Bolivariana Seccional Bucaramanga \\ Correo electrónico: macieltorrespsicologa@gmail.com
}

Lilibeth Mejía Jaimes

Psicóloga

Especialista en Salud Ocupacional y Riesgos Laborales Universidad Pontificia Bolivariana Seccional Bucaramanga

Correo electrónico: lillymejia2601@gmail.com

Carlos Arturo Conde Cotes

Médico

PhD. en Ciencias con énfasis en Neurofisiología Universidad Industrial de Santander Correo electrónico: cconde@uis.edu.co

\section{Silvia Botelho de Oliveira}

Psicóloga

PhD. en Ciencias con énfasis en Psicobiología Universidad Pontificia Bolivariana Seccional Bucaramanga

Recibido: $11 / 03 / 2019$

Evaluado: 01/04/2019

Correo electrónico: silvia.botelho@upb.edu.co

\section{Resumen}

El objetivo del presente estudio fue identificar comorbilidad entre TEPT y TDM en personas víctimas del desplazamiento forzado y factores asociados. Se realizó una investigación de enfoque cuantitativo, diseño no experimental de corte transversal y alcance descriptivo-analítico. Fueron evaluadas 120 personas, de ambos géneros, entre 18 a 57 años, seleccionadas a través de un muestreo no probabilístico por conveniencia. Se utilizó una entrevista clínica estructurada para diagnósticos del Eje I (SCID-I) basada en los criterios del DSM IV para el TEPT y TDM, posterior a la aplicación de un cuestionario de datos sociodemográficos y antecedentes clínicos con el que se registraron los factores de riesgos y síntomas asociados. La prevalencia para TEPT fue del 48\%, para TDM del 32\% y 21\% para comorbilidad, asociada con ideación e intento suicida y síntoma de rumiación. Los resultados fueron discutidos a la luz de otros estudios de prevalencia y la importancia de los factores de riesgo asociados en la comprensión de la comorbilidad entre TEPT y TDM.

Palabras clave Desplazamiento por la violencia, Trastorno por Estrés Postraumático, Trastorno Depresivo Mayor, Comorbilidad, Factores de Riesgo.

9 Para citar este artículo: Torres-Salazar, Y.M., Mejía-Jaimes, L., Conde-Cotes, C.A. y Botelho-De Oliveira, S. (2021). Víctimas del desplazamiento forzado: comorbilidad entre trastorno por estrés postraumático (TEPT) y depresivo mayor (TDM). Informes Psicológicos, 21(1), 133-149 http://dx.doi.org/10.18566/infpsic.v21n1a09 


\section{Post-traumatic Stress Disorder and Major Depressive Disorder in the context of displacement due to violence}

Abstract

The objective of this study was to identify comorbidity between PTSD and MDD in people who are victims of forced displacement and associated factors. A research with a quantitative approach, a nonexperimental cross-sectional design and a descriptive-analytical scope was carried out. 120 people, of both genders, from 18 to 57 years old, selected through a non-probabilistic convenience sampling, were evaluated. A structured clinical interview for Axis I diagnoses (SCID-I) was used based on the DSM IV criteria for PTSD and MDD, after the application of a questionnaire of sociodemographic data and clinical history with which the factors of risks and associated symptoms were registered. The prevalence for PTSD was $48 \%, 32 \%$ for MDD, and $21 \%$ for comorbidity, associated with suicidal ideation and attempt and rumination symptoms. The results were discussed in light of other prevalence studies and the importance of associated risk factors in understanding the comorbidity between PTSD and MDD.

\section{Keywords}

displacement by violence, Post-Traumatic Stress Disorder, Major Depressive Disorder, comorbidity, risk factors..

\section{Transtorno de estresse pós-traumático e transtorno depressivo maior no contexto de deslocamento devido à violência \\ Resumo}

0 objetivo deste estudo foi identificar a comorbidade entre TEPT e TDM em pessoas vítimas de deslocamento forçado e fatores associados. Realizou-se uma pesquisa com abordagem quantitativa, delineamento transversal não experimental e escopo analítico-descritivo. Foram avaliadas 120 pessoas, de ambos os sexos, com idade entre 18 e 57 anos, selecionadas por meio de amostra não probabilística de conveniência. A entrevista clínica estruturada para diagnósticos do Eixo I (SCID-I) foi utilizada com base nos critérios do DSM IV para TEPT e TDM, após a aplicação de um questionário de dados sociodemográficos e história clínica com os fatores de riscos e sintomas associados. A prevalência de TEPT foi de 48\%, 32\% para TDM e 21\% para comorbidade, associada a ideação suicida e sintomas de tentativa e ruminação. Os resultados foram discutidos à luz de outros estudos de prevalência e da importância dos fatores de risco associados no entendimento da comorbidade entre TEPT e TDM.

Palavras chave Deslocamento por violência, Transtorno de Estresse Pós-Traumático, Transtorno Depressivo Maior, comorbidade, fatores de risco. 


\section{ntroducción}

Según el Registro Único de Víctimas (RUV) del Gobierno de Colombia, son víctimas del desplazamiento forzado aquellas personas que manifestaron en su declaración, ser victimizadas por hechos en el marco del conflicto armado en Colombia. Según esta misma fuente, actualmente existe un total de $\mathbf{8 . 4 0 0 . 8 5 6}$ personas víctimas del conflicto armado en Colombia, de las cuales 152.770 en el Departamento de Santander y 48.954 en Bucaramanga.

En el caso de los conflictos armados, el miedo a la violencia se manifiesta como sentimientos de inseguridad y amenaza que de manera crónica afecta la salud mental y física durante y después del conflicto (Williams, Ghimire, \& Snedkerc, 2018). Estudios realizados con víctimas revelan que son personas altamente susceptibles de presentar diferentes tipos de afectaciones psicológicas, entre ellas, ansiedad, alteraciones del estado de ánimo y síntomas de estrés postraumático (HewittRamírez et al., 2016; Le, Tracy, Norris, \& Galea, 2013) tales como sentimientos de temor y miedo generados por los eventos traumáticos y el mismo desplazamiento, los cuales determinan politrauma y TEPT (Alejo, 2005; Campo-Arias, Sanabria, Ospino, Guerra, \& Caamaño, 2017).

Según el DSM-5 $5^{\mathrm{TM}}$ este trastorno es "secundario a exposición a la muerte, lesión grave o violencia sexual, ya sea real o amenaza" (p. 162). Se caracteriza por la presencia de recuerdos y sueños angustiantes relacionados con el suceso traumático, alteraciones negativas cognitivas y del estado de ánimo, reacciones disociativas, malestar psicológico y evitación experiencial de estímulos asociados a los eventos traumáticos, una estrategia adaptativa dirigida a evitar pensamientos, conductas y recuerdos asociados al evento traumático (Bados, 2015; Pickett, Bardeen, \& Orcutt, 2011; DSM-5'TM 2014). Por otro lado, como consecuencia de las grandes pérdidas, tanto materiales como de seres queridos (Moya, \& Figuerola, 2011), suelen sufrir un proceso de duelo, que puede evolucionar a un episodio Depresivo Mayor (García, 2012; HerreraLopez, \& Cruzado, 2014), el cual se caracteriza por la presencia de irritabilidad, tristeza, llanto, angustia, anhedonia, ideas de muerte e intentos o planes suicidas (DSM-5 ${ }^{\mathrm{TM}}$, 2014).

Algunos estudios realizados con víctimas del desplazamiento forzado en Colombia han revelado una prevalencia del 21\% del TEPT en Bucaramanga y el área metropolitana (Alejo, Rueda, Ortega, \& Orozco, 2007), 45.2\% en Sincelejo, Sucre (Echenique, Medina, Medina, \& Ramírez, 2008) y del $97.27 \%$ en Ciudad Bolívar, Bogotá (Sinisterra, Figueroa, Moreno, Robayo, \& Sanguino, 2010; Tamayo et al., 2016. En relación al Trastorno Depresivo Mayor se ha encontrado una prevalencia de $18.2 \%$ en lbagué y áreas rurales (Sánchez, Casas, Grais, Hustache, \& Moro, 2009), 30\% en el Cairo, Valle del Cauca y $41 \%$ en Medellín, Antioquia (Richards et al., 2011).

Considerando la condición de vulnerabilidad a que están expuestas, las personas víctimas del desplazamiento forzado pueden desarrollar y/o mantener, de manera temporal, uno o más trastornos a la vez, lo que, según la Organización Mundial de la Salud (OMS, 1995), se define como 
comorbilidad. El TEPT presenta comorbilidad con varios trastornos psiquiátricos, entre ellos, con el TDM (Ducrocq, \& Jehel, 2014; Elhai et al., 2015), ya que ambos pueden ser precipitados por un factor etiológico común como la exposición al estrés y la disminución de experiencias placenteras (Dzib-Goodin, Jiménez, Estévez, \& Sanders, 2016; Kashdan, Elhai, \& Frueh, 2007). Varios autores han sugerido que la comorbilidad TEPT-TDM se asocia a la coexistencia del estado de ánimo depresivo (disforia), que en el caso del TEPT se relaciona al síntoma de angustia no especificada (Contractor et al., 2014). Además, se ha destacado que la depresión es el trastorno más frecuente y el que está más asociado con los síntomas del trauma en víctimas del desplazamiento forzado, identificando la co-ocurrencia de trastornos afectivos y síntomas relevantes del TEPT (Alejo et al., 2007; Gómez-Restrepo et al., 2016).

Una gran preocupación relacionada con la comorbilidad entre el TEPT y TDM consiste en que ésta aumenta el riesgo de intento suicida (Arenson et al., 2018; Gómez-Restrepo et al. 2016; Oquendo, Brent, \& Birmaher, 2005), los cuales están vinculados a sentimientos de derrota, atrapamiento, tristeza, desesperanza (Panagioti, Gooding, Taylor, \& Tarrier, 2013). Por otro lado, ambos trastornos comparten el síntoma anhedónico, lo que genera preocupación, dada la relación estrecha entre anhedonia e ideación suicida (Ballard et al., 2017; Xie et al., 2014).

Considerando que la presencia de un trastorno aumenta significativamente la probabilidad de que otros se presenten (Londoño, Sicachá, \& González, 2011) y que la coexistencia entre TEPT-TDM aumenta el riesgo de comportamiento suicida (Ballard et al., 2017; Xie et al., 2014), el objetivo del presente estudio fue identificar la prevalencia de TEPTTDM y los potenciales factores asociados en una muestra de personas víctimas del desplazamiento forzado, que asistieron al Centro de Atención Integral a Víctimas (CAIV) de la ciudad de Bucaramanga-Santander.

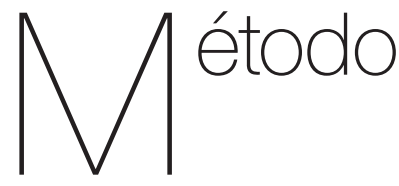

Se realizó un estudio cuantitativo, observacional de corte transversal y alcance descriptivo-analítico puesto que se pretendió describir la prevalencia del TEPT, TDM y de comorbilidad TEPT-TDM, identificando algunos factores potencialmente asociados.

\section{Participantes}

La muestra estuvo conformada por 120 personas víctimas del desplazamiento forzado que asistieron al CAIV de Bucaramanga durante el mes de septiembre de 2015. Fueron excluidos los participantes analfabetos, con problemas visuales y auditivos no corregidos, enfermedades neurológicas o reporte de tratamiento farmacológico (psiquiátrico o neurológico) o de consumo de psicofármacos de abuso en el momento de la evaluación. Los participantes fueron seleccionados utilizando un muestreo no probabilístico por conveniencia. 


\section{Instrumentos}

\section{Cuestionario de datos sociodemográficos y antecedentes clínicos.}

Mediante un cuestionario diseñado para el estudio se indagó el sexo, la edad, el estrato socioeconómico y el nivel de escolaridad. Adicionalmente, se exploraron datos importantes sobre la salud física y psicológica de los participantes, con el fin de identificar el cumplimiento o no de los criterios de inclusión requeridos por el proceso de investigación los cuales consistieron en ser víctima del desplazamiento forzado según la selección hecha por el CAIV, con un rango de edad entre 18 a 57 años de edad y de exclusión como ser analfabetas, presentar problemas visuales y auditivos no corregidos, estar en tratamiento farmacológico (psiquiátrico o neurológico), reporte de enfermedades neurológicas o psiquiátricas e informar de consumo de psicofármacos de abuso en el momento de la evaluación.

Entrevista Clínica Estructurada para Diagnósticos del Eje I (SCID-I) basada en los criterios diagnósticos del DSM-IV para TEPT (SCID-TEPT; First, Spitzer, Gibbon, \& Williams, 1999).

Compuesto por 24 ítems que corresponden a la sección F39 a la F64 del SCID-I en el cual se responde Si o No de acuerdo a la interpretación del entrevistado. La puntuación positiva para todos los criterios (A, B, C, D, E, y F) refiere diagnóstico positivo para el TEPT. De esta entrevista se registraron la presencia de eventos traumáticos como abuso sexual, presenciar un homicidio, ideación e intento de suicidio, experiencia de duelo y violencia sexual, con el fin de identificar cuáles de estas variables podían ser consideradas como factores asociados a la comorbilidad entre TEPT y TDM.

Entrevista Clínica Estructurada para Diagnósticos del Eje I (SCID-I) basada en los criterios diagnósticos del DSM-IV para TDM (SCID-TDM; First, Spitzer, Gibbon, \& Williams, 1999).

Compuesto por 15 ítems que corresponden a la sección A1 a la A15 del SCID-I. En la tabla de calificaciones se cumplen los criterios A, C, D, y E, en la cual, un puntaje positivo para todos estos criterios se establece el TDM.

\section{Procedimiento}

Se llevó a cabo un contacto inicial con el CAIV de la Alcaldía Municipal de Bucaramanga. El proceso de evaluación se realizó en un período de cuatro semanas durante el mes de septiembre de 2015, evaluándose en promedio 6 a 7 personas por día en un tiempo estipulado de una hora y media a dos horas por cada sesión. Se aplicó el cuestionario de datos sociodemográficos y antecedentes clínicos, el cual permitió evaluar el cumplimiento de los criterios de inclusión en el estudio; posteriormente se solicitó la firma del consentimiento informado y se realizaron las entrevistas estructuradas en una secuencia aleatoria para evitar sesgos de aplicación. 


\section{Análisis de los datos}

Las características de los participantes se analizaron mediante estadística descriptiva acorde con la naturaleza y distribución de las variables incluidas en el estudio. La comparación de la prevalencia de condición clínica, según los eventos y síntomas, se evaluó aplicando un test de $\chi^{2}$. Finalmente, se exploraron posibles asociaciones entre los síntomas y eventos con cada una de las tres condiciones clínicas (TEPT, TDM y TEPT+TDM) aplicando modelos de regresión binomial simple, con el fin de estimar las Razones de Prevalencia (RP) y sus intervalos de confianza del 95\%. Todo el análisis consideró un $\alpha$ de .05 y fue realizado en el software Stata 14,2.

\section{Consideraciones éticas}

El estudio se realizó de acuerdo con los principios éticos de investigación con humanos y es considerado de riesgo mínimo según el artículo 11 del apartado $B$ de la Resolución 8430 de 1993 (Ministerio de Salud, República de Colombia, 1993) y la Declaración de Helsinki, el cual fue avalado por el comité de ética de la Universidad Pontificia Bolivariana, Seccional Bucaramanga. El proyecto fue realizado en el marco de la práctica profesional de las estudiantes de la Facultad de Psicología de la Universidad Pontificia Bolivariana, Seccional Bucaramanga, en el segundo semestre de 2015, siguiendo los lineamientos éticos de investigación y práctica profesional.

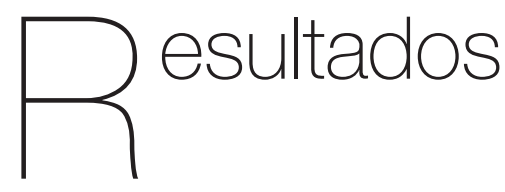

En relación con las variables sociodemográficas, se evaluaron 120 víctimas de desplazamiento forzado con un promedio de edad de 42.3 \pm 10.1 años, 63 mujeres (52.5\%) con edad promedio de

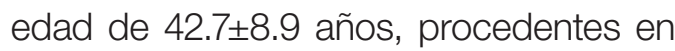
su mayoría del departamento de Santander (63.33\%), primordialmente del estrato $1(87.5 \%)$ y nivel de escolaridad primaria (61.7\%) (Tabla 1).

Tabla 1.

Características sociodemográficas de la muestra (n:120).

\begin{tabular}{llcc}
\hline \multirow{2}{*}{ Variable } & Categoría de la Variable & Frecuencia & Porcentaje de Participación \\
\hline \multirow{2}{*}{ Sexo } & Femenino & 63 & 52.5 \\
& Masculino & 57 & 47.5 \\
\hline \multirow{4}{*}{ Sepantander } & 76 & 63,3 \\
& Cesar & 14 & 11.7 \\
& Norte de Santander & 10 & 8.3 \\
& Antioquia & 5 & 4.2 \\
& Bolivar & 3 & 2.5 \\
& Otros & 12 & 9.9 \\
\hline
\end{tabular}




\begin{tabular}{llcc}
\hline Variable & Categoría de la Variable & Frecuencia & Porcentaje de Participación \\
\hline \multirow{3}{*}{ Estrato } & 0 & 3 & 2.5 \\
& 1 & 105 & 87.5 \\
& 2 & 11 & 9.7 \\
& 3 & 1 & .83 \\
\hline \multirow{3}{*}{ Escolaridad } & Primaria & 74 & 61.7 \\
& Secundaria & 45 & 37.5 \\
& Universitaria & 1 & .8 \\
\hline
\end{tabular}

En la Tabla 2 se observa que la prevalencia del TEPT fue del $48.3 \%$ (57\% en mujeres) mientras que la del TDM fue del $31.7 \%$ (36.5\% en mujeres). Por otro lado, la comorbilidad entre TEPT y el TDM en las personas víctimas del desplazamiento forzado fue del $20.8 \%$ (22.2\% en mujeres).

Tabla 2.

Prevalencia de TEPT, TDM y Comorbilidad (TEPT + TDM) en la población desplazada por la violencia según sexo (n:120).

\begin{tabular}{|c|c|c|c|c|c|c|c|c|c|}
\hline \multirow{2}{*}{ Variable } & \multicolumn{3}{|c|}{ TEPT } & \multicolumn{3}{|c|}{ TDM } & \multicolumn{3}{|c|}{ TEPT + TDM } \\
\hline & $\mathrm{n}$ & Prev. & IC95\% & $\mathrm{n}$ & $(\%)$ & IC95\% & $\mathrm{n}$ & $(\%)$ & IC95\% \\
\hline Mujeres & 36 & 57.1 & $44.9-69.4$ & 23 & 36.5 & $24.6-48.4$ & 14 & 22.2 & $12-32.5$ \\
\hline Hombres & 22 & 38.6 & $26-51.2$ & 15 & 26.3 & $14.9-37.7$ & 11 & 19.3 & $9.1-29.5$ \\
\hline Total & 58 & 48.3 & $39.4-57.3$ & 38 & 31.7 & $23.3-40$ & 25 & 20.8 & $13.6-28.1$ \\
\hline
\end{tabular}

Nota: Prevalencia/100 e intervalos de confianza al 95\% (IC95\%) de los trastornos de estrés post-traumático (TEPT), del trastorno depresivo mayor (TDM) y de la comorbilidad del TEPT Y TDM.

En la Figura 1 se encuentra la distribución de diagnósticos en cada síntoma o evento para las tres condiciones clínicas analizadas. Cabe señalar que la totalidad de quienes presentaron duelo correspondió las personas con TEPT; de otro lado, entre quienes fueron víctimas de actos relacionados con violencia sexual, el 50\% correspondió a diagnósticos de TDM y el restante a comorbilidad. Para los demás síntomas o eventos, el
TEPT se encontró entre 36.7\% y 56.7\%, correspondientes a intento/idea suicida y presenciar asesinatos, respectivamente. La frecuencia más baja fue para TDM en la mayoría de los síntomas y, en comparación la comorbilidad estuvo entre $25 \%$ y $50 \%$ en el registro de intento suicida y violencia sexual, también respectivamente. Cabe señalar que estas asociaciones no fueron estadísticamente significativas $(p=.06)$. 


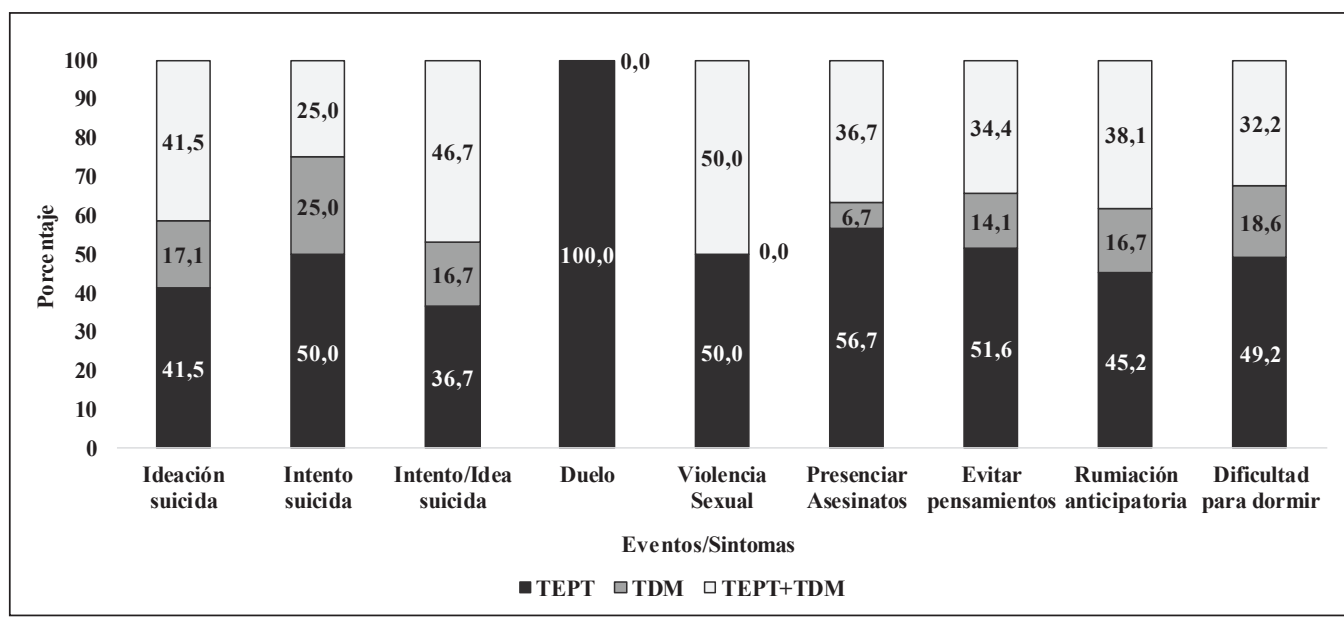

Figura 1. Distribución de frecuencia de eventos y síntomas asociados a TEPT, TDM y Comorbilidad (TEPT + TDM) en la población desplazada por la violencia.

Los hallazgos del análisis de regresión binomial mostraron asociaciones positivas y estadísticamente significativas entre el género femenino (RP 1,48), duelo $(2,2)$, presenciar asesinatos (RP 1,35), evitar pensamientos (RP 6,1), rumiación anticipatoria $(\mathrm{RP} 1,80)$ y dificultad para dormir (RP $2,22)$ con la presencia de TEPT. En cuanto a los factores asociados con TDM se encontraron como significativos la edad $\geq 45$ años (RP 1,89) y la rumiación anticipatoria (RP 1,81). A su vez, la violencia sexual se asoció también de manera significativa y positiva con la comorbilidad (RP 2,59). Cabe señalar que la ideación suicida, el intento ideación y la rumiación anticipatoria se asociaron positiva y significativamente con las tres condiciones clínicas (Tabla 3).

Tabla 3.

Asociación entre las variables sociodemográficas, eventos y síntomas registrados con los trastornos de estrés posttraumático (TEPT), del trastorno depresivo mayor (TDM) y la comorbilidad (TEPT + TDM) en personas víctimas de desplazamiento forzado.

\begin{tabular}{|c|c|c|c|c|c|c|c|c|c|}
\hline \multirow{2}{*}{$\begin{array}{c}\text { Características Sociodemográficas } \\
\text { y Eventos asociados }\end{array}$} & \multicolumn{3}{|c|}{ TEPT } & \multicolumn{3}{|c|}{ TDM } & \multicolumn{3}{|c|}{ TEPT+TDM } \\
\hline & $\mathrm{RP}$ & IC95\% & $\mathrm{p}$ & $\mathrm{RP}$ & IC95\% & $\mathrm{p}$ & $\mathrm{RP}$ & IC95\% & $\mathrm{p}$ \\
\hline Género Femenino & 1.48 & $1.0-2.19$ & .049 & 1.39 & $.81-2.39$ & .24 & 1.15 & $0.57-2.34$ & .69 \\
\hline Edad $\geq 45$ & .96 & $.66-1.39$ & .84 & 1.89 & $1.09-3.3$ & .02 & 1.96 & $0.94-4.09$ & .07 \\
\hline Estrato 0 y 1 & 1.5 & $.66-3.41$ & .33 & 2.0 & $0.55-7.3$ & .29 & 2.67 & $0.39-18.0$ & .31 \\
\hline Escolaridad primaria & 1.1 & $.74-1.61$ & .65 & 1.52 & $.84-2.77$ & .16 & 1.32 & $0.62-2.81$ & .47 \\
\hline Ideación suicida & 1.85 & $1.27-2.70$ & .001 & 2.24 & $1.29-3.89$ & .004 & 2.78 & $1.30-5.93$ & .008 \\
\hline Intento suicida & 1.61 & $1.03-2.52$ & .035 & 1.65 & $.78-3.48$ & .191 & 1.22 & $0.35-4.27$ & .76 \\
\hline Intento/Ideación & 1.63 & $1.15-2.32$ & .006 & 2.16 & $1.30-3.58$ & .003 & 2.75 & $1.38-5.47$ & .004 \\
\hline Duelo & 2.2 & $1.66-2.92$ & $<.0001$ & - & & & - & & \\
\hline Violencia Sexual & - & & & 1.63 & $.70-3.80$ & .26 & 2.59 & $1.07-6.27$ & .035 \\
\hline
\end{tabular}




\begin{tabular}{|c|c|c|c|c|c|c|c|c|c|}
\hline \multirow{2}{*}{$\begin{array}{c}\text { Características Sociodemográficas } \\
\text { y Eventos asociados }\end{array}$} & \multicolumn{3}{|c|}{ TEPT } & \multicolumn{3}{|c|}{ TDM } & \multicolumn{3}{|c|}{ TEPT+TDM } \\
\hline & $\mathrm{RP}$ & IC95\% & $\mathrm{p}$ & $\mathrm{RP}$ & IC95\% & $p$ & $\mathrm{RP}$ & IC95\% & $\mathrm{p}$ \\
\hline Evitar pensamientos & 6.1 & $2.06-18.1$ & .001 & 1.48 & $.73-3.0$ & .28 & 2.44 & $0.79-7.59$ & .122 \\
\hline Rumiación anticipatoria & 1.80 & $1.22-2.64$ & .003 & 1.81 & $1.05-3.12$ & .032 & 2.10 & $1.00-4.37$ & .047 \\
\hline Dificultad para dormir & 2.22 & $1.27-3.90$ & .005 & 1.74 & $.88-3.42$ & .11 & 1.47 & $.64-3.38$ & .37 \\
\hline
\end{tabular}

Nota: (RP) Razones de Prevalencia, (IC95\%) Intervalos de Confianza del 95\%, (P) probabilidad.

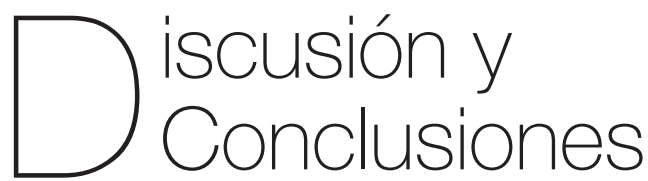

De manera general, se encontró una mayor prevalencia de TEPT (48\%) que de TDM (32\%), coherente con un sin número de eventos estresores a los que han sido expuestas las personas víctimas del desplazamiento forzado. Estos resultados concuerdan con aquellos revelados por Sarmiento (2016) demostrando, por medio de diagnósticos de salud mental realizados según el sistema de clasificación de los trastornos mentales o DSM IV, que la población de desplazados representó un mayor riesgo de desarrollar TEPT (el doble y seis veces más comparado con la población vulnerable sin aseguramiento y la población asegurada, respectivamente).

Hallazgo similar fue reportado en el estudio de Andrade (2008) realizado también con familias en situación de desplazamiento forzado reasentadas en el municipio del Cairo, quien evidenció, utilizando la Escala de Trauma Davidson (DTS) que mide la frecuencia y gravedad de los síntomas del TEPT, que el 100\% de la población presentó indicadores de TEPT, con una gravedad extrema del $50 \%$ y moderada del $40 \%$ y depresión grave de $30 \%$ con la escala de depresión de Zung. Estos indicadores tan elevados del TEPT, podrían deberse al número de traumas vivenciados (Choi, 2019) y a la severidad de los mismos, dado que son factores determinantes en el desarrollo de este trastorno (Van Zyl, Oosthuizen \& Seedat, 2008). Adicionalmente, a la proximidad de las evaluaciones, las cuales fueron realizadas 6 meses después del evento vital traumático.

A favor de lo anterior, Alejo et al. (2007) evidenciaron un mejoramiento gradual de los síntomas con relación al tiempo de desplazamiento, lo cual, según estos autores, es consistente con la remisión en el caso del paso de estrés agudo a crónico, y la remisión total en cerca del $40 \%$ de las personas expuestas. En este estudio, fueron evaluados 851 adultos en 15 asentamientos distribuidos en cuatro municipios del área metropolitana de Bucaramanga, encontrando una prevalencia del 21\% del TEPT con una versión en español de la entrevista estructurada Clinician Administered PTSD Scale for DSMIV (CAPS) y cumplimiento de los criterios diagnósticos de TEPT según el DSM-IV, usando el protocolo SCID.

La mayoría de los estudios publicados al respecto han revelado indicadores de prevalencia igualmente elevados, como el realizado en Ciudad Bolívar, Bogotá, donde, utilizando la Escala Estructurada Breve para el Diagnóstico de TEPT, se 
encontró una prevalencia del $97.27 \%$ del TEPT (Sinisterra et al., 2010). Asimismo, en Sincelejo, Sucre, se observó una prevalencia del $45.2 \%$, identificándose por medio del Cuestionario de Experiencias Traumáticas TQ y la Escala para Estrés Postraumático administrada por el clínico que los sucesos violentos que causaron el desplazamiento fueron los mismos que desencadenaron el TEPT (Echenique et al., 2008). Utilizando la Checklist (Pineda, Guerrero, Pinilla \& Estupiñán, 2002), Richards et al. (2011) encontraron una prevalencia de TEPT (88\%) y TDM (41\%) en una muestra similar a la evaluada en el presente estudio. Estos autores realizaron un estudio mixto en el cual se evaluaron los relatos de los participantes por medio de un auto-informe, realizando un análisis cualitativo también de los datos. La mayoría de los participantes eran mujeres las cuales, según Mogollón-Pérez, y Vázquez- Navarrete (2006), narran con mayor facilidad un evento traumático y suelen ser las más afectadas por los cambios en los roles familiares producto del desplazamiento.

Por el contrario, Sánchez et al. (2009) arrojaron una mayor prevalencia del TDM (18.2\%) en comparación con el TEPT (8.4\%). No obstante, este estudio posee características muy diferentes, puesto que la muestra estuvo conformada por niños y adultos de lbagué, pertenecientes a áreas rurales y urbanas que habían presenciado eventos traumáticos. Asimismo, era de corte longitudinal, por lo que durante tres años la población que evaluaron estuvo recibiendo una atención psicológica por medio de psicoterapia; y las personas que presentaron TDM, específicamente los pacientes que presentaban dificultades para ejecutar actividades básicas diarias, ideas suicidas y alteraciones significativas de la conciencia, recibieron medicación psicotrópica.

En la presente investigación se encontró una prevalencia de comorbilidad entre TEPT y TDM del $21 \%$ en personas víctimas del desplazamiento forzado, coherente con la reportada en otros estudios (Oquendo et al., 2005; Serrano, Ortiz, \& Bohórquez, 2009). Algunos investigadores han reportado indicadores más altos donde los participantes que presentaron TEPT, también fueron clasificados con sintomatología del TDM y trastorno de ansiedad (Flory \& Yehuda, 2015; Johansen, Eilertsen, Nordanger, \& Weisaeth, 2012). Según Johansen et al. (2012), la exposición a la violencia física ocasionada por diferentes motivos, podría explicar un nivel alto de prevalencia del TEPT, TDM y trastorno de ansiedad, y la comorbilidad entre ellos.

Algunos científicos han propuesto que la co-ocurrencia de ambos trastornos después de un evento traumático representa un constructo de estrés postraumático general (Elhai et al., 2011), mientras que otros sugieren que son constructos independientes (Gros, Simms, \& Acierno, 2010; Post, Feeny, Zoellner, \& Connell, 2016; Post, Zoellner, Youngstrom, \& Feeny, 2011), haciendo referencia a la importancia de los afectos negativos y vulnerabilidad en el TEPT y TDM. Según este abordaje, el TEPT está asociado a un miedo de sensaciones físicas mientras que el TDM está asociado a una baja afectividad positiva.

Otros investigadores han sugerido que la comorbilidad entre TEPT y TDM responde a dificultades diagnósticas, considerando que los criterios del DSM se han ido modificando en diferentes versiones 
para el caso del TEPT, pero no para el TDM. Asimismo, la presencia simultánea de TEPT y TDM podría representar un fenotipo relacionado con el trauma que es distinto del TDM (Flory \& Yehuda, 2015). En conjunto, estos estudios sugieren que comprender la co-ocurrencia de ambos trastornos podría ser útil para determinar si la presencia de ambos trastornos requiere un enfoque terapéutico diferente (Elhai et al., 2011; Flory, \& Yehuda, 2015; Gros et al., 2010), promoviendo la elaboración de tratamientos más acordes (Post et al., 2016).

Importante resaltar que el TEPT y TDM se asocian a un factor etiológico común que es la exposición al estrés (Kashdan et al., 2007) y comparten sintomatologías como la anhedonia (Ballard et al., 2017), el consumo de sustancias psicoactivas y la dependencia alcohólica (Cambra \& Camarillo, 2015). Acorde con lo anterior, el síntoma de rumiación se asoció positiva y significativamente con las tres condiciones clínicas evaluadas, mientras que evitar pensamientos y la dificultad para dormir se asociaron, específicamente, con el TEPT. Estos resultados refuerzan los hallazgos de que los pensamientos de orientación negativa como la rumiación podrían explicar la comorbilidad entre los diferentes trastornos emocionales (González, lbáñez, \& Barrera, 2017; Kalmbach, Pillai \& Ciesla, 2016). Estudios enfocados en este síntoma podrían aportar datos relevantes para la comprensión de comorbilidad entre TEPT y TDM.

La prevalencia de cada uno de los trastornos y de comorbilidad fue mayor en las mujeres. Estos resultados coinciden con otros estudios (Andrade, 2008; Richards et al., 2011). Posiblemente por su condición de vulnerabilidad debido a que en su mayoría son mujeres cabeza de familia que perdieron su pareja o fueron abandonadas por ellas en el momento del desplazamiento asumiendo roles antes invisibles y respondiendo por el cuidado de su núcleo familiar (Auto 092 del 2008; Mogollón-Pérez \& Vázquez-Navarrete, 2006). Adicionalmente, las mujeres víctimas están expuestas a diferentes factores de riegos, entre ellos y acorde con el presente estudio, se ha demostrado que aquellas expuestas a relaciones sexuales violentas presentan un mayor riesgo de padecer TDM y TEPT, en comparación con aquellas mujeres víctimas de otras formas de abuso (García-Toro, Rubio, Gili, Roca, Jin, Liu et al., 2013; Wiesel, \& Markus, 2013). Se ha demostrado también que en el primer mes después de sufrir abuso sexual, el $43 \%$ de las mujeres evaluadas presentaron TEPT moderado o muy severo y el 52.2\% depresión moderada o severa, los cuales se redujeron a los seis meses (Machado et al., 2011). Todos estos hallazgos concuerdan con los del presente estudio que demuestran que la exposición a violencia sexual aumenta aproximadamente tres veces la probabilidad de que las personas padezcan de comorbilidad.

La asociación encontrada entre duelo y el TEPT en el presente estudio coinciden con los revelados por Rheingold, Byczkiewicz, Saunders y Kilpatrick (2011) quienes encontraron que las personas que han perdido a alguien por homicidios resultaban ser más propensas a presentar TEPT que las víctimas de otro tipo de violencia. Dicha asociación podría ser consecuencia a la exposición de las victimas del desplazamiento forzado a pérdidas significativas como el abandono de sus tierras o la muerte de sus seres queridos, conllevando a un proceso de duelos múltiples que involucra inestabilidad 
emocional, social y psicológica, deterioro en la capacidad para expresar sentimientos y evitación de todo aquello que tenga relación con el pasado (Alejo, 2005; Moya \& Figuerola, 2011).

La ideación e intento suicida resultaron ser factores de riesgo para presentar TEPT, TDM o comorbilidad TEPT-TDM. Estos resultados concuerdan con los encontrados en diferentes estudios donde se demostró que la comorbilidad incrementa el riesgo de ideación suicida (Arenson et al., 2018; Bantjes, Phil, Kagee, McGowan, \& Steel, 2016; Panagioti, Gooding, \& Tarrier, 2012). Algunas teorías han propuesto que la tendencia suicida en el TEPT está asociada con sentimientos de derrota, atrapamiento, tristeza y desesperanza (Panagioti et al. 2012), siendo las percepciones de derrota y atrapamiento los procesos que subyacen los pensamientos suicidas e importantes predictores de la conducta suicida (Panagioti, Gooding, \& Tarrier, 2015). Considerando que los individuos que presentan comorbilidad entre TEPT y TDM muestran síntomas más severos que cuando sufren de uno o de otro trastorno (Post et al., 2011; Post et al., 2016), se podría esperar una mayor susceptibilidad de presentar TEPT, TDM o comorbilidad aquellos que reportaron ideación suicida.

En conjunto, el presente estudio aporta datos relevantes para la realización de estudios enfocados a la comprensión de la comorbilidad existe entre TEPT y TDM, así como a la elaboración de intervenciones acordes y encaminadas a mejorar la salud de las personas víctimas del desplazamiento forzado, los cuales están siendo considerados en la creación de un protocolo de atención dirigido específicamente a esta población.

\section{Agradecimientos}

A la Dirección de Investigaciones y Transferencia de la Universidad Pontificia Bolivariana de Bucaramanga, por el apoyo financiero (Proyecto \#098-0116-3100). A todas las personas en condición de desplazamiento por la violencia del área metropolitana de Bucaramanga que participaron voluntariamente en el estudio.

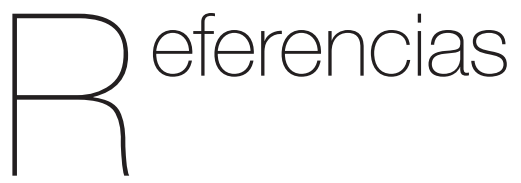

Alejo, E. (2005). Aplicación del modelo de estrés postraumático en el estudio del impacto de la violencia sobre la salud mental en población desplazada. MedUnab, 8(1), 23-28.

Alejo, E., G., Ortega, M., \& Orozco, L. (2007). Estudio epidemiológico del Trastorno por Estrés Postraumático en población desplazada por la violencia política en Colombia. Universitas Psychologica, 6(3), 623-635.

Andrade, J. (2008). Efectos psicopatológicos del conflicto armado colombiano en familias en situación de desplazamiento forzado reasentadas en el municipio del Cairo en el año 2008. Orbis. Revista Científica Ciencias Humanas, 7(20), 111-114.

Arenson, M.B., Whooley, M.A., Neylan, T.C., Maguen, S., Metzler, T.J., \& Cohen, B.E (2018). Posttraumatic stress disorder, depression, and suicidal ideation inveterans: Results from the mind your heart study. 
Psychiatry Research, 26, 224-230. https:// doi.org/10.1016/j.psychres.2018.04.046

American Psychiatric Assoc American Psychiatric Association., Kupfer, D. J., Regier, D. A., Arango López, C., AyusoMateos, J. L., Vieta Pascual, E., \& Bagney Lifante, A. (2014). DSM-5: Manual diagnóstico y estadístico de los trastornos mentales (5a ed.). Madrid [etc.]: Editorial Médica Panamericana.

Bados, A. (2015). Trastorno por Estrés Postraumático. Facultat de Psicologia, Departament de Personalitat, Avaluació i Tractament Psicològics, Universitat de Barcelona. Recuperado de http://diposit. ub.edu/dspace/bitstream/2445/65623/1/ TEPT.pdf

Ballard, E. D., Wills, K., Lally, N., Richards, E. M., Luckenbaugh, D. A., Walls, T., Ameli, R., Niciu, M. J., Brutsche, N. E., Park, L., \& Zarate, C. A. (2017). Anhedonia as a clinical correlate of suicidal thoughts in clinical ketamine trials. Journal of Affective Disorders, 218, 195-200. doi: 10.1016/j. jad.2017.04.057

Bantjes, J.R., Phil, D., Kagee, A., McGowan, T., \& Steel, H. (2016). Symptoms of posttraumatic stress, depression, and anxiety as predictors of suicidal ideation among South African university students. Journal of American College Health, 64(6), 429-437. https://doi.org/10.1080/07448481 .2016 .1178120

Cambra, J., \& Camarillo, L. (2015). Trastornos de ansiedad relacionados con traumas y otros factores de estrés. MedicinePrograma de Formación Médica Continuada Acreditado, 11(84), 4999-5007. doi: 10.1016/j.med.2015.07.009
Campo-Arias, A., Sanabria, A. R., Ospino, A., Guerra, V. M., \& Caamaño, B. H. (2017). Polivictimización por el conflicto armado y sufrimiento emocional en el departamento del Magdalena, Colombia. Revista Colombiana de Psiquiatría, 46, 147-153. doi: 10.1016/j.rcp.2016.06.005

Choi, J.Y. (2019). Predictors of the cooccurrence of posttraumatic stress disorder and depressive disorder in psychiatric outpatients. Comprehensive Psychiatry, 89, 40-45. https://doi.org/10.1016/j. comppsych.2018.12.005

Contractor, A., Durham, T., Brennan, J., Armour, C., Wutrick, H., Frueh, C., \& Elhai, J. (2014). DSM-5 PTSD's symptom dimensions and relations with major depression's symptom dimensions in a primary care sample. Psychiatry Research, 215(1), 146-153. doi: 10.1016/j.psychres.2013.10.015

Ducrocq, F., \& Jehel, L. (2014). Trastornos psicotraumáticos. Tratado de Medicina, 18(3), 1-7. doi: 10.1016/ S1636-5410(14)68151-4

Dzib-Goodin, A., Jiménez, E. K., Estévez, R., \& Sanders, L. (2016). Sistemas biológicos involucrados en el trastorno de estrés post traumático. Revista Mexicana de Neurociencia, 17(5), 83-97.

Echenique, C., Medina, L., Medina, A., \& Ramírez, A. (2008). Prevalencia del trastorno por estrés postraumático en población desplazada por violencia, en proceso de restablecimiento en Sincelejo. Psicología desde el Caribe. Universidad del Norte, 21, 122-135.

Elhai, J. D., Carvalho, L. F., Miguel, F. K., Palmieri, P. A., Primi, R., \& Frueh, B. C. (2011). Testing whether posttraumatic 
stress disorder and major depressive disorder are similar or unique constructs. Journal of Anxiety Disorders, 25, 404-410. doi:10.1016/j.janxdis.2010.11.003

Elhai, J.D., Contractor, A.A., Tamburrino, M., Fine, T.H., Cohen, G., Shirley, E., \& Galea, S. (2015). Structural relations between DSM5 PTSD and major depression symptoms in military soldiers. Journal of Affective Disorders, 175, 373-378 .doi.org/10.1016/j. jad.2015.01.034

First, M., Spitzer, R., Gibbon, M., \& Williams, J. (1999). Guía del usuario para la Entrevista Clínica Estructurada para los Trastornos del Eje I del DSM-IV. MASSON, S.A.

Flory, J.D \& Yehuda, R. (2015). Comorbidity between post-traumatic stress disorder and major depressive disorder: alternative explanations and treatment considerations. Dialogues in Clinical Neuroscience, 17(2), 141-150.

García, H. (2012). La depresión etiológica y tratamiento. Ciencia y Sociedad, 37(2), 183-197.

García-Toro, M., Rubio, J.M., Gili, M., Roca, M., Jin, C.J., Liu, C.J. et al. (2013). Persistence of chronic major depression: A national prospective study. Journal of Affective Disorders 151, 306-312. doi: 10.1016/j. jad.2013.06.013

Gómez-Restrepo, C., Tamayo-Martínez, N., Buitrago, G., Guarnizo-Herreño, C. C., Garzón-Orjuela, N., Eslava-Schmalbach, J., ... \& Rincón, C. J. (2016). Violencia por conflicto armado y prevalencias de trastornos del afecto, ansiedad y problemas mentales en la población adulta colombiana. Revista Colombiana de
Psiquiatría, 45(S1), 147-153. doi: 10.1016/j. rcp.2016.11.001

González, M., Ibáñez, I. \& Barrera, A. (2017). Rumiación, preocupación y orientación negativa al problema: procesos transdiagnósticos de los trastornos de ansiedad, de la conducta alimentaria y del estado de ánimo. Acta Colombiana de Psicología, 20(2), 30-41. http://www.dx.doi. org/10.14718/ACP.2017.20.2.3

Gros, D. F., Simms, L. J., \& Acierno, R. (2010). Specificity of posttraumatic stress disorder symptoms: An investigation of comorbidity between posttraumatic stress disorder symptoms and depression in treatmentseeking veterans. Journal of Nervous and Mental Disease, 198, 885-890. doi: 10.1097/NMD.0b013e3181fe7410

Herrera-Lopez, V., \& Cruzado, L. (2014). Estrés postraumático y comorbilidad asociada en víctimas de la violencia política de una comunidad campesina de Huancavelica, Perú. Revista de Neuropsiquiatría, 77(3), 144-159. doi: 10.20453/rnp.2014.2029

Hewitt-Ramírez, N., Juárez, F., Parada-Baños, A. J., Guerrero-Luzardo, J., RomeroChávez, Y. M., Salgado-Castilla, A. M., \& Vargas-Amaya, M. V. (2016). Afectaciones psicológicas, estrategias de afrontamiento y niveles de resiliencia de adultos expuestos al conflicto armado en Colombia. Revista Colombiana de Psicología, 25(1), 125-140. doi: 10.15446/rcp.v25n1.49966

Johansen, A., Eilertsen, D., Nordanger, D., \& Weisaeth, L. (2012). Prevalence, comorbidity and stability of posttraumatic stress disorder and anxiety and depression symptoms after exposure to physical assault: An 8-year prospective longitudinal study. Nordic Journal of 
Psychiatry, 67(1), 69-80. doi: 10.3109/ 08039488.2012 .732112

Kalmbach, D. A, Pillai, V., \& Ciesla, J. A. (2016). The correspondence of changes in depressive rumination and worry to weekly variations in affective symptoms: $\mathrm{A}$ test of the tripartite model of anxiety and depression in women. Australian Journal of Psychology, 68(1), 52-60. DOl: https://doi. org/10.1111/ajpy.12090.

Kashdan, T., Elhai, J., \& Frueh, C. (2007). Anhedonia, emotional numbing, and symptom overreporting in male veterans with PTSD. Personality and Individual Differences, 43, 725-735. doi: 10.1016/j. paid.2007.01.013

Le, F., Tracy, M., Norris, F., \& Galea, S. (2013). Displacement, county social cohesion, and depression after a large-scale traumatic event. Social Psychiatry and Psychiatric Epidemiology, 48, 1729-1741. doi:10.1007/ s00127-013-0698-7

Londoño, N., Sicachá, M., \& González, J. (2011). Posibles manifestaciones del trastorno por estrés postraumático en adultos desplazados por el conflicto armado del asentamiento "Acacias Bajo" en Armenia Quindío. Sinapsis, 3(3), 172- 185.

Machado, C.L., de Azevedo, R.C., Facuri. C.O., Vieira. M.J., \& Fernandes, A.M. (2011). Posttraumatic stress disorder, depression, and hopelessness in women who are victims of sexual violence. The International Journal of Gynecology \& Obstetrics, 113(1), 58-62. doi: 10.1016/j.jjgo.2010.10.016

Mogollón-Pérez, A.S., \& Vázquez- Navarrete, M.L. (2006). Opinión de las mujeres desplazadas sobre la repercusión en su salud del desplazamiento forzado. Gaceta Sanitaria, 20(4), 260-265.

Moya, M., \& Figuerola, M. (2011). El duelo, más allá del dolor. Desde el Jardín de Freud, 11, 133-148.

Oquendo, M., Brent, D., \& Birmaher, B. (2005). Posttraumatic stress disorder comorbid with major depression: factors mediating the association with suicidal behavior. The American Journal of Psychiatry, 162(3), 560-566. $\quad$ https://doi.org/10.1176/appi. ajp.162.3.560

Panagioti, M., Goodinga, P.A., \& Tarrier, N. (2012). A meta-analysis of the association between posttraumatic stress disorder and suicidality: the role of comorbid depression. Comprehensive Psychiatry, 53(7), 915-930. https://doi.org/10.1016/j. comppsych.2012.02.009

Panagioti, M., Goodinga, P.A, \& Tarrier, N. (2015). A prospective study of suicidal ideation in posttraumatic stress disorder: the role of perceptions of defeat and entrapment. Journal of Clinical Psychology, 71(1), 50-61. doi: 10.1002/jclp.22103

Panagioti, M., Gooding, P., Taylor, P., \& Tarrier, N. (2013). A model of suicidal behavior in posttraumatic stress disorder (PTSD): The mediating role of defeat and entrapment. Psychiatry Research, 209(1), 55-59. doi: 10.1016/j.psychres.2013.02.018

Pickett, S., Bardeen, J., \& Orcutt, H. (2011). Experiential avoidance as a moderator of the relationship between behavioral inhibition system sensitivity and posttraumatic stress symptoms. Journal of Anxiety Disorders, 25(8), 1038-1045. doi: 10.1016/j.janxdis.2011.06.013 
Pineda, D., Guerrero, O., Pinilla, M., \& Estupiñán, M. (2002). Utilidad de un cuestionario para rastreo del estrés postraumático en una población colombiana. Revista de Neurología, 34(10), 911-916.

Post, L. M., Feeny, N.C., Zoellner, L.A., \& Connell, A.M. (2016). Post-traumatic stress disorder and depression co-occurrence: Structural relations among disorder constructs and trait and symptom dimensions. Psychology and Psychotherapy: Theory, Research and Practice, 89, 418-434. doi:10.1111/ papt.12087

Post, L. M., Zoellner, L. A., Youngstrom, E., \& Feeny, N. C. (2011). Understanding the relationship between co-occurring PTSD and MDD: Symptom severity and affect. Journal of Anxiety Disorders, 25, 11231130. doi:10.1016/j.janxdis.2011.08.003

Rheingold, A., Byczkiewicz, M., Saunders, B., \& Kilpatrick, D. (2011). Examining Posttraumatic Stress Symptoms in a National Sample of Homicide Survivors: Prevalence and Comparison to Other Violence Victims. Journal of Traumatic Stress, 24(6), 743-746. doi:10.1002/ jts.20692

Richards, A., Ospina, J., Barrera, M., Escobar, J., Ardila, M., Metzler, T., \& Mamar, C. (2011). Posttraumatic stress disorder, anxiety and depression symptoms, and psychosocial treatment needs in Colombians internally displaced by armed conflict: A mixed method evaluation. Psychological Trauma: Theory, Research, Practice, and Policy, 3(4), 384-393. doi: 10.1037/a0022257

Rytwinski, N.K., Scur, M.D., Feeny, N.C., \& Youngstrom, E.A. (2013). The cooccurrence of major depressive disorder among individuals with posttraumatic stress disorder: a meta-analysis. Journal of Traumatic Stress, 26(3), 299-309.

Sánchez, E., Casas, G., Grais, R., Hustache, S., \& Moro, R. (2009). The Colombian conflict: a description of mental health program in the Department of Tolima. Conflict Health 3(13), 1-6. doi: 10.1186/1752-1505-3-13.

Sarmiento, R. (2016). Trastorno de estrés postraumático, ansiedad y depresión en adolescentes y adultos expuestos al conflicto armado en Colombia 2005-2008. MEDICINA (Bogotá), 38(2), 134-156.

Serrano, A., Ortiz, D., \& Bohórquez, A. (2009). Frecuencia de las comorbilidades psiquiátricas del eje I en los pacientes con diagnóstico de trastorno por estrés postraumático. Revista Med, 17(1), 20-25.

Sinisterra, M., Figueroa, F., Moreno, V., Robayo, M., \& Sanguino, J. (2010). Prevalencia del trastorno de estrés post traumático en población en situación de desplazamiento en la localidad de Ciudad Bolívar Bogotá, Colombia 2007. Psychologia: Avances de la Disciplina, 4(2), 83-97.

Tamayo, N., Rincón, C. J., De Santacruz, C., Bautista, N., Collazos, J., \& GómezRestrepo, C. (2016). Problemas mentales, trastornos del afecto y de ansiedad en la población desplazada por la violencia en Colombia, Resultados de la Encuesta Nacional de Salud Mental 2015. Revista Colombiana de Psiquiatría, 45(1), 113-118. http://dx.doi.org/10.1016/j.rcp.2016.09.004

Van Zyl, M., Oosthuizen, P.P. \& Seedat, S. (2008). Posttraumatic stress disorder: undiagnosed cases in a tertiary inpatient setting. African Journal of Psychiatry, 11, 119-122. 
Wiesel, R., \& Markus, L. (2013). Perception vs. Circumstances of the Child Sexual Abuse Event in Relation to Depression and Posttraumatic Stress Symptomatology. Journal of Child Sexual Abuse, 22, 519533. doi: 10.1080/10538712.2013.800932

Williams, N.E., Ghimire, D. \& Snedkerc, K.A. (2018). Fear of violence during armed conflict: Social roles and responsibilities as determinants of fear. Social Science Research, 71, 145-159. https://doi. org/10.1016/j.ssresearch.2018.01.004
Xie, W., Li, H., Luo, X., Fu, R., Ying, X., Wang, N., ... \& Shi, C. (2014). Anhedonia and pain avoidance in the suicidal mind: behavioral evidence for motivational manifestations of suicidal ideation in patients with major depressive disorder. Journal of ClinicalPsychology, 70(7), 681-692. doi: 10.1002/jclp.22055 